Mots. Les langages du politique

\title{
La police nationale au défi des relations presse. Une information sous contrôle?
}

Guillaume Le Saulnier

\section{(2) OpenEdition \\ Journals}

Édition électronique

URL : https://journals.openedition.org/mots/20700

DOI : $10.4000 /$ mots. 20700

ISSN : 1960-6001

Éditeur

ENS Éditions

Édition imprimée

Date de publication : 15 septembre 2012

Pagination : 129-142

ISBN : 9782847883732

ISSN : 0243-6450

Référence électronique

Guillaume Le Saulnier, « La police nationale au défı des relations presse. Une information sous contrôle? ", Mots. Les langages du politique [En ligne], 99 | 2012, mis en ligne le 15 septembre 2014, consulté le 22 avril 2022. URL : http://journals.openedition.org/mots/20700 ; DOI : https://doi.org/ $10.4000 /$ mots. 20700

\section{(C) ENS Éditions}




\section{La police nationale au défi des relations presse. Une information sous contrôle?}

La police nationale est omniprésente dans les médias, tant dans le registre de l'information que dans celui du divertissement. Pourtant, cette publicité intensive ne va pas de soi pour une institution dépositaire de la force publique, fonctionnellement tournée vers le secret et soucieuse de sa réputation. Elle intègre de nombreux enjeux. À un premier niveau, la police trouve dans les médias une tribune providentielle pour promouvoir ses services, ses activités et ses résultats, et ce jusque dans les fictions grand public ${ }^{1}$. Cependant, le foisonnement de ses apparitions médiatiques rend d'autant plus nécessaire mais difficile le maintien d'une image publique cohérente et positive. À un degré supérieur, les médias représentent une arène politique où se joue une lutte permanente pour définir la réalité et prescrire les orientations de l'action publique. Les journalistes remplissent une fonction de vigilance critique sur la politique policière et, plus spécifiquement, sur les usages de la force publique. Ils permettent notamment l'intervention de tiers extérieurs, qui viennent concurrencer les décideurs administratifs dans la gestion de la sécurité publique (Thœnig, 1994).

Dans les termes de l'analyse stratégique (Crozier, Friedberg, 1977), les médias constituent une «incertitude cruciale » pour l'institution policière. On peut alors s'interroger sur les stratégies que celle-ci mobilise pour en conjurer les menaces et en exploiter les opportunités. Cette question a déjà été formulée en 1993 dans un numéro des Cahiers de la sécurité intérieure consacré aux relations police/médias : "Le ministère de l'Intérieur a-t-il une politique de communication?»(Roger, Zamponi, 1993). Elle mérite un examen actualisé, intégrant la généralisation et la professionnalisation de la communication, constituée comme une activité spécialisée, avec ses doctrines, ses enjeux et ses techniques. À partir des années 1980, celle-ci s'étend du secteur privé aux administrations publiques, pour s'imposer comme une «nouvelle forme ou catégorie de l'action de l'État» (Ollivier-Yaniv, 1997, p. 80). Désormais, les institutions publiques ne veulent plus subir mais tourner à leur avantage le principe

1. Voir «Go Fast: pourquoi le ministère de l'Intérieur a “coproduit” le film », Mediapart, 4 octobre 2008.

Université de Reims Champagne-Ardenne, CEREP / GREMTOS guillaume.le-saulnier@univ-reims.fr 
de publicité. Pour cela, un outil privilégié réside dans les relations presse. Ce syntagme désigne une gestion rationalisée des rapports avec les journalistes, tournée vers la coopération, en vue de convertir l'information en promotion. Pour la sociologie de la communication, il renvoie à des procédures internes de contrôle de la parole publique : on entend par là une distribution instituée du pouvoir de parler, mais aussi des procédés de «lissage» discursif, à savoir "un système de contraintes qui fixe les contours de l'énonciation légitime» (Oger, Ollivier-Yaniv, 2006, p. 66) pour en garantir la continuité et la cohérence.

Dès lors, on questionnera à nouveau la politique de communication de la police nationale : celle-ci est-elle partie prenante ou restée en marge de l'extension de la communication? Quel est le degré d’intégration et de formalisation des relations presse dans la force publique? On placera au cœur de notre interrogation la capacité de l'institution policière à produire une parole publique «disciplinée», à la fois coordonnée et cohérente. Plus précisément, il s'agit d'identifier les mécanismes (formels et informels) censés intégrer les instances énonciatives et réguler leurs prises de parole, mais aussi de sonder les limites de ces procédures de contrôle.

Pour cela, on exploitera un stage «en immersion» (selon les termes officiels) effectué en 2008 pendant trois mois dans une Direction départementale de la sécurité publique (DDSP), au sein des services actifs de la police urbaine, et dans une préfecture, tous lieux situés dans un département de la région parisienne. Nous avons procédé à dix-sept entretiens semi-directifs individuels auprès de responsables et de syndicalistes policiers mandatés (à des degrés divers) pour administrer les relations presse, mais aussi auprès du directeur de cabinet du préfet et de son chargé de communication. Ces récits de pratique ont été complétés par des entretiens auprès de deux journalistes locaux, respectivement à l'Agence France Presse et au Parisien, et d'un journaliste de la presse magazine spécialisé dans la force publique. Nous avons également recueilli les circulaires ministérielles et les documents internes relatifs à l'organisation et au fonctionnement de la communication policière. Ce double matériau (discursif et textuel) permet d'évaluer la correspondance et, inversement, les frottements ou les écarts entre les consignes prescrites et les pratiques effectives ou du moins déclarées. Il est enrichi par des observations situées sur des actions de communication, qui constituent autant de cas empiriques permettant de compléter ou de corriger le travail d'analyse.

Par sa durée et ses matériaux combinés, cette enquête de terrain fournit un accès de première main aux univers de sens et aux manières de faire en matière de relations presse. Sur cette base, on étudiera leur organisation et leur fonctionnement de l'intérieur, dans un système hautement bureaucratisé, au moyen d'une sociologie compréhensive. On procédera en trois étapes: d'abord, on explicitera les obstacles théoriques et empiriques rencontrés sur pareil sujet et leurs implications méthodologiques; ensuite, on démontrera 
l'intégration en demi-teinte des relations presse, entre volontarisme et dissuasion; enfin, on dévoilera les tensions et les contradictions contenues dans cette activité, qui engendrent des distorsions dans la parole publique policière.

\section{Carences théoriques et effets de censure}

Paradoxalement, au regard de l'omniprésence médiatique de la police nationale et des enjeux de cette visibilité, et par contraste avec des travaux anglosaxons précoces et nombreux, la communication policière reste un sujet délaissé dans la recherche française en sciences sociales. Pourtant, dès 1980, une première impulsion est donnée au Centre d'études et de recherches sur la police (CERP) de l'université Toulouse 1, avec une thèse dirigée par le politologue Jean-Louis Loubet del Bayle; elle est suivie vingt ans plus tard par une thèse sur le traitement médiatique des violences policières (alléguées ou avérées) dans la presse quotidienne nationale (Loubet del Bayle, Cubaynes, 1988; Yebouet Boah Cofy, 2001). Entre-temps, l'Institut des hautes études sur la sécurité intérieure (IHESI), fondé en 1989 à l'initiative du ministre de l'Intérieur Pierre Joxe, consacre un numéro de la revue Les Cahiers de la sécurité intérieure aux relations police/médias. Mais, hormis ces travaux pionniers, la communication policière n'est traitée qu'en ordre dispersé et comme partie satellitaire d'articles ou d'ouvrages, notamment dans la sociologie du journalisme et des problèmes publics².

On mentionnera deux obstacles théoriques pour expliquer une telle carence. Le premier réside dans le « média-centrisme» (Schlesinger, 1992), sous ses multiples variantes : péché originel de la sociologie des médias, il consiste à restreindre leur étude à une observation des conditions et des produits du travail journalistique. Or, on sait que le rapport aux sources est un déterminant majeur de la fabrication des informations, en raison de la dépendance des journalistes à leurs informateurs et de la sophistication croissante des stratégies de communication utilisées par les acteurs extérieurs au champ médiatique pour influencer la teneur et l'issue du débat public. Le second tient à l'emprise de deux thèses symétriquement opposées. D'un côté, inspirée par la théorie critique, la thèse de la collusion réduit la police et les médias à un instrument du pouvoir destiné à contrôler les «masses», suivant un couplage entre coercition physique et répression symbolique (Marcuse, 2001). D’un autre côté, portée par les policiers et les journalistes, la thèse de l'antagonisme insiste sur les contradictions fonctionnelles et les rivalités professionnelles qui opposent les deux métiers, pour figer ceux-ci dans une position d'ennemis héréditaires (Huberson, 1993).

2. Voir notamment : Charon, Furet, 2000, p.155-172 ; Bonelli, 2001; Mucchielli, 2001, p. 26-54; Macé, Peralva, 2002 ; Marchetti, 2008, p. 93-129. À noter également, sur la publicité consacrée aux violences policières : Jobard, 2002, p. 113-178; Dewerpe, 2006. 
À cet égard, on rappellera le dernier article de la «charte des devoirs professionnels des journalistes français» de 1918, stipulant qu' «un journaliste digne de ce nom [...] ne confond pas son rôle avec celui du policier»3.

Contre une telle polarisation, on insistera sur les liens anciens et routiniers entre les policiers et les journalistes. En effet, l'institution d'une force publique suppose un contrôle structurel sur ses activités; or, les médias représentent, en tant que "quatrième pouvoir », un rouage essentiel de cette vigilance démocratique. En outre, la constitution historique des faits divers comme genre informatif, de même que le triomphe du journalisme d'investigation à partir des années 1980, sont inséparables des liens noués entre les journalistes et leurs informateurs policiers (Courcol etal., 1993). En termes fonctionnalistes, ces liens étroits procèdent d'une dépendance mutuelle : les policiers sont une source privilégiée, en tant que détenteurs légitimes et souvent exclusifs des informations policières, tandis que les journalistes sont des intermédiaires obligés, au sens où ils contrôlent l'accès à la publicité. Dès lors, le modèle pertinent pour décrire leurs relations est celui de la négociation entre «associés-rivaux» (Tunstall, cité par Neveu, 2004, p. 60), amenés à coopérer sur la base de leurs intérêts réciproques. On ajoutera que l'omniprésence médiatique de la police permet de prendre à contre-pied la tradition critique : celle-ci définit la force publique comme «l'œil du pouvoir », à savoir une institution répressive et intrusive, dont la fonction panoptique consiste à tenir sous le regard le corps social pour maintenirl'ordre (Foucault, 1975); or, elle est autant sinon plus l'objet que le sujet d'une visibilité intensive et invasive, dès lors qu'elle se trouve projetée sur le devant de la scène médiatique et placée sous le regard et le débat publics.

Ces obstacles théoriques sont redoublés par les difficultés de l'enquête de terrain. L'entretien fournit un accès privilégié aux systèmes de représentation, en tant que savoir élaboré par les acteurs sociaux sur un segment de leur existence (Blanchet et al., 2000). Mais il se heurte aux dispositions activées par les policiers dans le cadre d'une interaction verbale sollicitée et dirigée par le chercheur. La première réside dans une méfiance instituée envers toute personne extérieure à la profession. Cette méfiance est renforcée par les conditions d'accès au terrain, puisque nous sommes "parachutés " par la hiérarchie centralisée dans les services déconcentrés, situation propice à attiser le soupçon sur les finalités de la recherche. La deuxième se manifeste par une propension à la justification : les policiers sont constamment amenés à justifier leurs actions auprès de la hiérarchie, de la tutelle judiciaire ou du public. Cette «pré-socialisation au rôle de répondant» (Hughes, 1996, p. 285) risque de convertir l'entretien en examen. La troisième tient au tropisme des violences policières : la grande majorité des enquêtés évoque d'emblée ce sujet polémique, supposé constitutif de toute investigation scientifique sur la force publique, pour pro-

3. Source: Syndicat national des journalistes (SNJ).

132 - Mots. Les langages du politique $n^{\circ} 99$ juillet 2012 
duire un discours de justification. Faute d'afficher une réserve prudente, sinon un mariage de raison avec les raisons de la police, il semble que le chercheur ne puisse revendiquer avec succès une approche compréhensive, voire se dépêtrer d'un doute tenace sur son manque de neutralité.

Nous avons constaté à nos dépens ces dispositions lors des entretiens exploratoires. Pour y remédier, nous avons renoncé à l'usage du dictaphone, suivant le conseil méthodologique de Dominique Monjardet (Ocqueteau, 2008, p. 275). En effet, puisque la police se caractérise par une «culture du secret», elle-même impulsée et renforcée par une «culture du soupçon » envers autrui (Monjardet, 1996), le dictaphone accentue les effets de censure et, à ce titre, il comporte un biais lourd. Il redouble l'injonction paradoxale contenue dans la situation d'entretien, qui sollicite une parole libre et spontanée. En outre, la présence d'un appareil destiné à enregistrer les faits de parole risque d'établir une analogie malencontreuse avec l'audition policière. En lieu et place, nous avons procédé à une prise de notes systématique, facilitée par la répétition d'un entretien à l'autre de termes clés permettant un codage de circonstance, et complétée par une réécriture immédiate pour restituer fidèlement le contenu verbal et non verbal de l'échange.

Un dernier obstacle empirique mérite d'être mentionné. À écouter la plupart des policiers, la communication policière serait balbutiante sinon inexistante : la force publique serait " pire que la grande muette »4, du moins elle ne saurait pas communiquer. Cette présomption de mutisme ou d'amateurisme témoigne de la distance sinon du divorce entre les exécutants, formellement tenus au silence 5 , et les porte-parole (hiérarchiques ou syndicaux) censés les représenter. Cependant, elle est rapidement démentie par le travail d'enquête et, à ce titre, elle procède soit d'une méconnaissance imputable à la profonde division du travail policier, soit d'un discours de façade produit par des professionnels de la ruse et des faux-semblants.

\section{Les relations presse sous emprise politique}

De nombreux indicateurs témoignent de l'intégration des relations presse dans la police nationale. Certes, cette intégration équivaut à une reprise en main «sur le tas et sur le tard ${ }^{6}$; elle vise à redorer le blason de la police vis-à-vis de la gendarmerie; surtout, elle est explicitement dirigée contre les syndicats policiers, constitués précocement comme des interlocuteurs privilégiés auprès des journalistes:

\footnotetext{
4. Entretien avec un responsable syndical.

5. Les fonctionnaires de police sont astreints au devoir de réserve, à l'obligation de discrétion, au secret professionnel et au secret de l'enquête et de l'instruction. De plus, ils ont interdiction de faire grève, outre un usage parcimonieux du droit de manifester.

6. Entretien avec un chef de service adjoint.
} 
Premièrement, c'est de culture dans la police de considérer que les journalistes font chier, et de nous plaindre ensuite que les gendarmes nous piquent la gloire avec une bonne communication. Deuxièmement, les syndicats policiers ont occupé le terrain de la communication pendant que les chefs de service ne communiquaient pas, ce qui a eu - parfois du moins - un impact catastrophique, c'est une vraie catastrophe pour l'administration policière! On a une nécessité de communiquer, on s'en rend compte, et progressivement même les plus rétifs sont incités à communiquer. Parce qu'on est sous une double pression : la communication de la gendarmerie et celle des syndicats.7

Le premier indicateur tient à la majoration des enjeux rattachés à la visibilité médiatique de la force publique. Les enquêtés évoquent les enjeux symboliques, en termes de notoriété et d'image, notamment pour les directions et les services de la sécurité publique. Mais ils insistent également sur les enjeux pratiques, au sens où les relations presse ont une incidence concrète. D'abord, dans une optique managériale, de nombreux chefs de service désignent les retombées presse comme un levier à part entière pour motiver leurs effectifs. Ensuite, ils attribuent à la communication policière une fonction informative, auprès du grand public, mais aussi une vertu dissuasive, quand elle manifeste l'efficacité de l'action policière :

Je pense que c'est toujours bon de montrer son travail : les médias sont un levier pour le management dans la fonction publique. Quand ils voient que leur travail est reconnu, les gars sont contents. En outre, ça nous permet de montrer à la population que la police bosse, qu'elle fait son boulot, ça permet de montrer les faits élucidés, ça peut même avoir un impact auprès des voyous. // Auprès des voyous? // Oui, ça peut avoir un effet dissuasif. Par exemple, on a eu une fraude avec la CPAM, on a décidé de communiquer pour que soit publié un article stratégique contre les fraudes. Donc ça permet de montrer qu'il y a des partenariats efficaces entre les institutions, et que ça paie. Le message, c'est qu'il n'y a pas d'impunité. ${ }^{8}$

En arrière-plan, cette conversion au jeu médiatique puise dans une conviction partagée quant aux effets directs et puissants des médias. D’ailleurs, leur « pouvoir» est théorisé dans la doctrine policière sur les violences urbaines, en tant que facteur essentiel de leur déclenchement et de leur propagation?. Enfin, les médias représentent une ressource potentielle pour influencer clandestinement le déroulement des procédures judiciaires, par des divulgations ou des «fuites» censées prendre à témoin l'opinion publique sur telle ou telle décision judiciaire.

Le second indicateur réside dans la politique de spécialisation en matière de relations presse, consolidée au début des années 2000 par une série de

7. Entretien avec un chef de service.

8. Idem.

9. Voir l'annexe intitulée «Mesure du rôle des médias» dans l'ouvrage testimonial de la commissaire Lucienne Bui Trong (2000, p. 171-174), sur son action à la Direction centrale des renseignements généraux (DCRG). 
notes internes et d'arrêtés ministériels. D'abord, elle suppose l'instauration, respectivement aux niveaux centralisé et déconcentré, de services destinés à piloter la communication ${ }^{10}$ et d' ' officiers de presse » mandatés pour gérer les relations presse. À ce titre, la récente désignation d'un porte-parole au côté du ministre de l'Intérieur n'est que la manifestation au sommet d'une évolution à tous les étages de la pyramide institutionnelle. Ensuite, cette spécialisation passe par une intégration des relations presse dans la formation initiale des commissaires et des officiers, complétée par des stages dédiés ("face caméra » et « ingénierie de la communication») pour les principaux intéressés, outre des conseils pratiques diffusés par intranet ${ }^{11}$. Enfin, elle va de pair avec la constitution d'une « doctrine », à travers des textes de référence où sont formalisés les finalités et les modes opératoires des relations presse, au premier rang desquels la «charte de la communication externe de la police nationale» datée du 10 septembre 2004. Cette charte, qui a valeur de circulaire ministérielle, se tourne résolument vers une stratégie offensive : il s'agit d'aller au-devant des médias, de faire preuve de réactivité et de réagir aux mises en cause en lieu et place d'un silence propice à la suspicion. Cela dit, elle recouvre également une fonction disciplinaire, au sens où elle insiste d'abord et avant tout sur la coordination des structures et des acteurs de la communication policière, pour en garantir la continuité et la cohérence. Cette priorité se traduit par une «chaîne de communication », selon une expression consacrée. Or, si les mots ont un sens, il s'agit bien plutôt d'une communication «enchaînée », tant sont nombreuses les restrictions qui en limitent et en dissuadent l'usage.

En effet, le volontarisme affiché en matière de relations presse cède le pas à une véritable obsession du contrôle. En premier lieu, la communication policière est soumise à un contrôle étroit et fébrile, exercé par la hiérarchie, le pouvoir politique, et la tutelle judiciaire pour les informations dont elle est le propriétaire légitime. Cette surveillance passe par une remontée systématique des informations: collectées par les services déconcentrés, celles-ci sont restituées à intervalles réguliers au préfet et au parquet par le directeur départemental de la sécurité publique, à travers une synthèse (dite "pochette») des données chiffrées, des «belles affaires » et des «faits marquants» retenus en fonction de leur intensité. Elle se matérialise également dans le contrôle a priori exercé sur les demandes de presse : les services déconcentrés ne peuvent y répondre que sur accord préalable du Service d'information et de communication de la police (SICOP) pour la Direction générale de la police nationale (DGPN), du Bureau de

10. À savoir la Délégation à l'information et à la communication (DICOM), en janvier 2004, et le Service d'information et de communication de la police (SICOP), en décembre 2005, respectivement au Secrétariat général du ministère de l'Intérieur et à la Direction générale de la police nationale (DGPN).

11. Notamment dans Le guide du communicant et le Guide de la communication en sécurité publique (non datés). 
la communication interministérielle $(\mathrm{BCl})$ pour la préfecture et éventuellement, du Secrétariat général du parquet pour la tutelle judiciaire.

En second lieu, la communication policière est hautement bureaucratisée, à travers deux mécanismes. Le premier est la centralisation du pouvoir décisionnel et des ressources, concentrés entre les mains de la haute hiérarchie et du corps préfectoral. À cet égard, la Direction départementale occupe une position subalterne vis-à-vis de la préfecture. De même, on notera la disproportion des moyens (matériels et humains) consacrés aux relations presse : l'État-major de la Direction départementale compte deux officiers de presse, contre quatre employés pour le $\mathrm{BCl}$ au cabinet du préfet, dont un responsable issu du secteur privé et spécialisé en communication. Le second mécanisme est la segmentation de la parole publique policière : dans les services déconcentrés, les officiers de presse et les chefs de service peuvent communiquer auprès de la presse locale sur les événements de routine, tandis que les médias nationaux, les événements de forte intensité et les informations à teneur polémique sont l'apanage exclusif de la préfecture, voire du ministère de l'Intérieur.

Pour toutes ces raisons, on peut parler d'une mise sous tutelle de la communication policière. Un tel confinement trouve son principe, pour beaucoup, dans le lien étroit entre la visibilité médiatique et l'exercice du pouvoir (Thompson, 2005) : en raison de leur rendement dans le jeu politique, tant pour les gouvernements successifs que pour leurs opposants, l'ordre et la sécurité sont désignés comme des sujets «sensibles » et les informations policières sont surveillées comme le lait sur le feu. Par exemple, en interne, les télégrammes relatifs aux violences urbaines portent souvent la mention «ne pas communiquer à la presse », tandis que les incendies de véhicules lors des fêtes nationales font l'objet d'une censure croissante ${ }^{12}$. C'est dire que la communication policière orchestrée par les services déconcentrés en direction de la presse locale procède d'une logique de dépolitisation : si les officiers de presse et les chefs de service sont tenus d'aller au-devant des journalistes pour leur «proposer régulièrement des sujets d'actualité ou de fond $»^{13}$, c'est moins pour satisfaire une quelconque exigence démocratique d'accountability que pour rabattre les informations policières sur des sujets factuels et des articles de complaisance. Les informations rendues publiques par le chef d'une circonscription de sécurité publique vont dans ce sens :

Je n'ai pas de contacts réguliers avec XXX [prénom d'un journaliste local], mais je vois un peu ce qui peut l'intéresser : par exemple, le jeune qui fume son pétard sous les fenêtres du commissariat, je l'ai appelé là-dessus, il a fait trois lignes dans

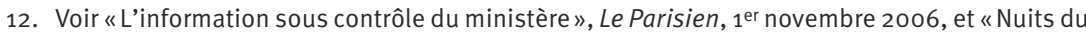
14-Juillet : la place Beauvau impose le silence sur le nombre de voitures incendiées », Le Monde, 18 juillet 2009.

13. Ministère de l'Intérieur, "Charte de la communication externe de la police nationale », 10 septembre 2004 . 
Le Parisien. Par contre, toutes les affaires plus grosses ou trop trash, je ne l'appelle pas, ce n'est pas toujours nécessaire, je privilégie plutôt les affaires croustillantes, et toutes celles qui peuvent valoriser le service. Je ne lui parle pas non plus des difficultés. ${ }^{14}$

Ce travail de dépolitisation, mené tous azimuts, trouve une illustration dans la communication de la préfecture sur le mouvement des travailleurs sanspapiers, survenu au printemps 2008. Sous l'égide du syndicat CGT, des demandes de régularisation sont déposées dans plusieurs préfectures, notamment en région parisienne. À cette occasion, une journaliste de LCI transmet au cabinet du préfet une demande pour filmer le dépôt des dossiers et interviewer le corps préfectoral. Le directeur de cabinet sollicite l'avis du ministère de l'Intérieur, avant de communiquer sur un sujet désigné comme « sensible». Un compromis est proposé à la journaliste : l'interview est refusée, mais le filmage est possible à condition de ne pas prendre le son, «pour éviter un coup tordu » 15 d'un militant de la CGT. Pour ne rien laisser au hasard, un policier en tenue est présent lors du dépôt des dossiers. Avant de filmer, la journaliste est reçue par le directeur de cabinet, pour « un briefing préalable sur un sujet sensible sur lequel un journaliste n'est pas forcément bien informé $»^{16}$. Le directeur de cabinet s'en tient aux éléments de langage définis par le ministère de l'Intérieur : il ne s'agit nullement d'une «régularisation massive» et les dossiers seront examinés «au cas par cas». À l'issue de cette séquence, il ne fait pas mystère de ses intentions réelles : s'il a répondu favorablement à cette demande de presse, c'est pour « revendiquer une image positive de transparence, et ne pas laisser dire que la préfecture a refusé de communiquer ${ }^{17}$. Ce faisant, il entend réduire la couverture télévisuelle du mouvement local à une "bonne image», en adéquation avec les standards journalistiques des chaînes d'information en continu.

\section{Une communication en désordre de bataille}

Les constats précédents suggèrent une communication policière disciplinée, mise en œuvre selon un canevas immuable. Pourtant, la police nationale ne parle pas d'une seule voix : le contrôle exercé sur la parole publique policière, aussi prégnant soit-il, est contrarié par les stratégies médiatiques utilisées tant par les services déconcentrés que par les syndicats policiers.

En premier lieu, pour les officiers de presse et les chefs de service, les relations presse tiennent de l'injonction paradoxale, entre incitations répétées et

14. Entretien avec un chef de service.

15. Entretien avec le responsable du $\mathrm{BCl}$.

16. Entretien avec le directeur de cabinet du préfet.

17. Idem 
règles dissuasives, exigence de réactivité et inertie bureaucratique. Une telle contradiction engendre des cercles vicieux bureaucratiques. Certains se protègent par une stratégie de sécurité : en respectant à la lettre les obligations formelles, ils utilisent celles-ci comme un «paravent » pour refuser toute communication ${ }^{18}$. À l'opposé, d'autres adoptent une stratégie de risque qui consiste à nouer de véritables alliances avec une poignée de journalistes locaux, en l'occurrence un correspondant de l'AFP et quelques journalistes du Parisien édition locale. Ces alliances supposent des liens stabilisés et éprouvés, consolidés par des services mutuels («donnant-donnant») et des gages de bonne volonté. De sorte que, pour l'essentiel, les relations presse se structurent par des liens personnalisés, où le sens moral est un critère omniprésent pour jauger et juger les journalistes dignes de confiance, par opposition aux «politicards» et aux «irresponsables».

Cette coopération intéressée équivaut à une «logique de monopole» (Crozier, Friedberg, 1977, p.169-174) : il s'agit de se constituer comme un interlocuteur privilégié, pour enfermer les médias dans une situation de dépendance et marginaliser les sources d'information concurrentes. Elle est favorisée par les conditions de travail des journalistes locaux, qui ne disposent guère des ressources nécessaires pour s'affranchir des sources officielles. Mais elle implique une contrepartie : pour fidéliser cette clientèle journalistique et se rendre difficilement substituable, les officiers de presse et les chefs de service sont tenus de lui fournir des informations exclusives, voire confidentielles. Par conséquent, cette stratégie instaure un contexte propice à un détournement des consignes prescrites, au profit des normes de circonstance élaborées à la faveur de ces liens personnalisés :

Par exemple, il y avait une série de vols sur des salariés qui vont et viennent de la gare. On a mis en place une surveillance, on a topé un mec, ensuite j'ai appelé XXX [prénom d'un journaliste local]. Je n'informe pas le maire systématiquement. J'aime bien une phrase de Talleyrand [récitant] : « On ne sort de l'ambiguïté qu'à ses dépens». Je ne préviens pas systématiquement la DDSP [Direction départementale de la sécurité publique], je devrais le faire si j'étais un bon soldat. Sur une grosse affaire, je le fais. ${ }^{19}$

Dans les termes de l'analyse stratégique, les relations presse débouchent sur la constitution d'un système d'action « relativement autonome dont la logique propre [est] irréductible à la finalité première et à la rationalité instrumentale qui ont présidé à sa naissance » (Crozier, Friedberg, 1977, p.176). Doté de ses propres enjeux et règles du jeu, ce système se superpose aux finalités et aux stratégies formellement assignées. Certes, ce dédoublement de la communication policière, entre une filière bureaucratisée et une filière personnali-

18. Entretien avec le responsable du SICOP.

19. Entretien avec un chef de service. 
sée, offre un regain de réactivité et une influence accrue sur les médias; mais il engendre une relative émancipation vis-à-vis de la hiérarchie et des tutelles politique et judiciaire et, partant, un moindre contrôle.

En second lieu, l'incitation sinon l'injonction à communiquer exacerbe la compétition interne pour l'accès aux médias, au détriment de l'impératif de coordination. En effet, certains responsables policiers sont tentés de profiter des «belles affaires» pour tirer à eux la couverture médiatique : celle-ci est synonyme de prestige, mais surtout elle est un moyen de renforcer leur position et celle de leur service à l'intérieur de l'organisation policière. De leur côté, les journalistes exploitent ces rivalités internes pour obtenir des informations, mais ils en sont également les instruments et parfois les otages. Par exemple, on mentionnera le faux pas d'un journaliste local auprès d'un responsable policier à la tête d'un commissariat : celui-ci est à l'origine d'une importante saisie de stupéfiants, mais elle est récupérée par un service départemental qui en profite pour "se faire mousser » sur la scène médiatique; pour regagner la confiance du chef de service lésé, le journaliste local se rachète peu après en publiant deux encadrés sur le démantèlement d'un trafic de stupéfiants dans sa circonscription.

En troisième lieu, cette dispersion de la communication policière est renforcée par l'activisme des syndicats policiers. Engagés de longue date sur le terrain médiatique, ceux-ci conçoivent les relations presse comme un levier à part entière pour renforcer leur position dans un champ syndical concurrentiel, mais aussi dans les rapports de force avec l'administration. Si leurs marges de liberté restent limitées, ils bénéficient d'une atténuation du devoir de réserve pour exprimer publiquement les doléances de la profession, en utilisant les médias comme une caisse de résonance. Deux thèmes sont particulièrement payants pour se frayer un accès à la publicité et faire voir et valoir les revendications catégorielles, à savoir le thème du «manque de moyens » et corrélativement celui du «malaise policier»:

On est là pour faire entendre la parole des collègues, le ressenti du terrain, le moral des collègues. Et les journalistes sont très friands de ça, tout ce qui est ressenti et moral des policiers, parce que ça leur permet de faire du sensationnel. // Ah oui? // Oui, ils nous demandent souvent des informations à ce sujet. Et ils nous contactent aussi pour des informations factuelles : textes, chiffres, etc. ${ }^{20}$

Cette insistance sur les difficultés de la profession va de pair avec la dénonciation de certaines décisions administratives. Dans le département étudié, un syndicat a obtenu le maintien des compagnies départementales d'intervention (CDI) en faisant reculer l'administration par un tapage médiatique ${ }^{21}$. Par cette fonction de politisation corporative, la communication syndicale fait

20. Entretien avec un délégué syndical.

21. Idem. 
obstacle à une gestion discrétionnaire de la force publique et, partant, elle s'oppose frontalement à la logique institutionnelle de dépolitisation. De plus, on observe une profonde cassure dans le travail de symbolisation de la force publique. En effet, les protestations syndicales sur « la police du pauvre et des pauvres» (Lenoir, 1993, p. 436) contrastent avec la promotion médiatique des «polices de pointe» (services spécialisés ou brigades d'intervention), utilisées par l'institution comme une vitrine pour réaffirmer sa puissance et sa compétence. Sous cet angle, on a affaire à des stratégies de communication divergentes sinon contradictoires, entre d'un côté une publicité doloriste de la profession, campée sur des témoignages prosaïques voire misérabilistes, et de l'autre des manifestations spectaculaires du pouvoir, à travers des sujets enchantés et des reportages embedded.

Malgré son ampleur et en dépit de la volonté ferme du pouvoir politique, la rationalisation des relations presse reste inachevée. Celles-ci sont prises dans une tension constante entre bureaucratisation et personnalisation, mais aussi entre dépolitisation institutionnelle et politisation corporative. Le double impératif de coordination et de cohérence au fondement de la communication policière achoppe, dans une large mesure, sur les marges de liberté exploitées par ses différents acteurs et sur la dispersion des stratégies mobilisées pour l'accès aux médias. Par son insistance sur les relations de pouvoir et les règles informelles engendrées par les systèmes bureaucratiques, l'analyse stratégique constitue donc une entrée pertinente pour dévoiler l'importance des contraintes organisationnelles dans l'interprétation et le détournement des consignes prescrites.

Plus largement, cette analyse de la parole publique policière recouvre une valeur heuristique pour l'étude de la communication des institutions publiques; c'est d'autant plus vrai que la police nationale représente, au-delà de ses particularismes, un «véritable laboratoire de l'administration» (Gleizal, 1993, p.67) pour les innovations de l'action publique. De nombreux travaux contestent toute verticale du pouvoir pour insister sur les conflits internes qui divisent les administrations et leur communication. Cette conflictualité se manifeste tout particulièrement lors des situations de crise, propices à un débordement des routines de travail instaurées entre les porte-parole et les journalistes et, partant, à la mise en concurrence de la version officielle avec des points de vue discordants (Lawrence, 2000). La présente analyse confirme et prolonge ce constat en démontrant l'existence de tensions, voire de contradictions internes, et ce jusque dans les stratégies d'autopromotion, c'est-àdire dans les relations presse en régime de routine. 


\section{Références}

Blanchet Alain, Ghiglione Rodolphe, Massonnat Jean, Trognon Alain, 2000 [1987], Les techniques d'enquête en sciences sociales. Observer, interviewer, questionner, Paris, Dunod.

BONELLI Laurent, 2001, « Renseignements généraux et violences urbaines », Actes de la recherche en sciences sociales, $\mathrm{n}^{0}$ 136-137, p. 95-103.

Bui TROng Lucienne, 2000, Violences urbaines. Des vérités qui dérangent, Paris, Bayard.

ChARON Jean-Marie, FurEt Claude, 2000, Un secretsi bien violé. La loi, le juge et le journaliste, Paris, Seuil.

Courcol Catherine et al., 1993, "Policiers et journalistes : entre compétition et connivence " (table ronde), Les Cahiers de la sécurité intérieure, nº12, p. 25-45.

Crozier Michel, Friedberg Erhard, 1977, L'acteur et le système. Les contraintes de l'action collective, Paris, Seuil.

DeWERPE Alain, 2006, Charonne, 8 février 1962. Anthropologie historique d'un massacre d'État, Paris, Gallimard.

Fou CAULt Michel, 1975, Surveiller et punir. Naissance de la prison, Paris, Gallimard.

GLEIZALJean-Jacques, 1993, La police en France, Paris, PUF.

HUbERSON Laurent, 1993, "Logique de la sécurité, logiques de l'information», Les Cahiers de la sécurité intérieure, $\mathrm{n}^{0} 12$, p. 47-55.

HuGHES Everett C., 1996, Le regard sociologique. Essais choisis, Paris, EHESS.

JoBARD Fabien, 2002, Bavures policières? La force publique et ses usages, Paris, La Découverte.

LAWrence Regina R., 2000, The Politics of Force. Media and the Construction of Police Brutality, Berkeley, University of California Press.

LENOIR Rémi, 1993, "Désordre chez les agents de l'ordre», P. Bourdieu éd., La misère du monde, Paris, Seuil, p. 421-492.

Loubet del BAyle Jean-Louis, Cubaynes Marie-Hélène, 1988, «La police et la presse : un système d'interactions », J.-L. Loubet del Bayle et al., Police et société, Toulouse, Presses de l'IEP de Toulouse / CERP, p. 149-175.

MACÉ Éric, Peralva Angelina, 2002, Médias et violences urbaines. Débats politiques et construction journalistique, Paris, La Documentation française / INHES.

Marchetti Dominique éd., 2008, Communication et médiatisation de l'État. La politique invisible, Grenoble, PUG.

MARCUSE Herbert, 2001, "Un nouvel ordre », Manière de voir, nº 56, p.10-12.

Monjardet Dominique, 1996, Ce que fait la police. Sociologie de la force publique, Paris, La Découverte.

Mucchielli Laurent, 2001, Violences et insécurité. Fantasmes et réalités dans le débat français, Paris, La Découverte.

NeVEu Éric, 2004, Sociologie du journalisme, Paris, La Découverte.

OCQUeTEAU Frédéric, 2008, "L'influence des travaux de Dominique Monjardet sur une nouvelle génération de chercheurs», D. Monjardet, Notes inédites sur les choses 
policières, 1999-2006. Suivi de : Le sociologue, la politique et la police, Paris, La Découverte, p. 269-280.

Oger Claire, Ollivier-Yaniv Caroline, 2006, «Conjurer le désordre discursif. Les procédés de "lissage" dans la fabrication du discours institutionnel», Mots. Les langages du politique, no 81, p. 63-77.

OLLIVIER-YANIV Caroline, 1997, «L'État communicant. Des formes de la communication gouvernementale», Quaderni, n³3, p. 75-83.

ROGER LUC, ZAMPONI Francis, 1993, "Le ministère de l'Intérieur a-t-il une politique de communication? », Les Cahiers de la sécurité intérieure, nº12, p. 65-69.

SCHLESINGER Philip, 1992, «Repenser la sociologie du journalisme. Les stratégies de la source d'information et les limites du média-centrisme », Réseaux, n 51, p. 75-98.

TH氏ENIG Jean-Claude, 1994, "La gestion systémique de la sécurité publique», Revue française de sociologie, vol. XXXV, nº 3, p. 357-392.

THOMPSON John B., 2005, «Transformation de la visibilité », Réseaux, n0100, p. 187-213.

Yebouet BoAh Cofy Pascal Henry, 2001, Presse et "affaires» policières en France de 1975 à 1995, thèse de science politique sous la direction de Jean-Louis Loubet del Bayle, Université Toulouse 1/ CERP, 2 tomes (document non publié). 